\title{
Sutureless double-patch-and-glue technique for repair of subacute left ventricular wall rupture after myocardial infarction
}

\author{
Francesco Alamanni, MD, Andrea Fumero, MD, Alessandro Parolari, MD, PhD, Piero Trabattoni, MD, \\ Aldo Cannata, MD, Gianluca Berti, MD, and Paolo Biglioli, MD, Milan, Italy
}

1 cute myocardial infarction is complicated by free wall rupture in about $5 \%$ of patients. Prompt surgical repair is the treatment of choice, even if operative mortality is sometimes exceedingly high (ie, 24\%-35\%). ${ }^{1}$ Several different surgical repairs have been proposed, including use of sutures with pledgets, Dacron patch, and infarctectomy, as well as use of biologically glued pericardial patches. ${ }^{2,3}$

We describe the use of a sutureless double-patch-and-glue technique for repair of this complication.

\section{Surgical Technique and Clinical Summary}

After anesthesia is induced, immediate median sternotomy and pericardiotomy are performed to relieve tamponade. Then the heart is cannulated in the standard fashion, the ascending aorta is clamped, antegrade crystalloid cardioplegic solution is given, and the ascending aorta and left atrial sumps are placed. The heart is dislocated by means of 2 tapes passed around the inferior vena cava and through the transverse sinus, and the infarcted area is inspected (Figure 1, A).

A first autologous pericardium patch is sized to be about 2.0 times greater with respect to the longer diameter of left ventricular discontinuity. Two to four 5-0 polypropylene stitches are placed as stay sutures to fix the patch to the epicardium toward the atrioventricular groove to the lesion and tied. About one third of the circumference of the patch is now fixed to the heart, and the remaining two thirds can be pulled up and down as a sail for the precise measurement of the relationship between the patch and the infarcted area. The epicardium and pericardial patch are carefully dried with an air blower, and then cyanoacrylate glue (Glubran; GEM Inc, Viareggio, Italy) is applied, starting from the part nearest to the atrioventricular groove. This glue transforms all the underlying epicardium and the myocardium into very stiff tissues. Perfect adherence among epicardium, glue, and patch is essential to avoid inhomogeneous distribution of the glue or lack of tension of the patch (Figure 1, B).

From the Department of Cardiac Surgery, University of Milano, Centro Cardiologico Monzino IRCCS, Milan, Italy.

Received for publication Feb 16, 2001; accepted for publication Feb 21, 2001.

Address for reprints: Alessandro Parolari, $\mathrm{MD}, \mathrm{PhD}$, Department of Cardiac Surgery, University of Milano, Centro Cardiologico Monzino IRCCS, Via Parea, 4, 20138, Milano, Italy (E-mail: aparolari@cardiologicomonzino.it or corallo@mailserver.unimi.it).

J Thorac Cardiovasc Surg 2001;122:836-7

Copyright (C) 2001 by The American Association for Thoracic Surgery

$0022-5223 / 2001 \$ 35.00+0 \quad \mathbf{1 2 / 5 4 / 1 1 5 4 1 5}$

doi: $10.1067 / \mathrm{mtc} .2001 .115415$
After that, a polyester or an etherologous pericardial patch is sized to be about 2 to 3 times greater than the first patch. Then the same steps previously described are followed to apply this second sail-like patch to the epicardium, and biologic bovine serum albumin and glutaraldehyde glue (BioGlue; CryoLife International, Inc, Kennesaw, Ga) are used. This glue, once polymerized, is elastic and does not theoretically interfere with the residual contractility of the perinfarcted myocardium covered only by this second patch (Figure 2, A). Before aortic clamp removal, the complete polymerization of this glue is verified (Figure 2, B). Then deairing procedures are performed, the aortic crossclamp is removed, and the patient is weaned from cardiopulmonary bypass (CPB), usually with the help of an intra-aortic balloon pump with a target mean arterial pressure of between 65 and $85 \mathrm{~mm} \mathrm{Hg}$ to avoid excessive stretching in the repair area.

With this technique, 3 patients underwent repair of postinfarction left ventricular lateral wall rupture. Aortic crossclamp and CPB times ranged from 22 and 35 minutes and 38 and 55 minutes, respectively. One patient underwent repair of postinfarction left ventricular lateral wall rupture with the same technique but without aortic crossclamping and CPB and with a beating heart because of the previous spontaneous tamponade of the bleeding. All the patients had an uneventful postoperative course and are alive at follow-up, which ranges from 3 to 22 months.

\section{Discussion}

Postinfarction left ventricular rupture usually results in massive hemorrhage into the pericardium, leading, in most cases, to rapid death before diagnosis and possible repair. ${ }^{4}$ The relatively few patients who reach the operating table alive represent a relatively rare course of subacute left ventricular free wall rupture, which is peculiar both because of an early diagnosis and because of the small size of the ventricular defect.

In these patients we propose a repair against the laws of physiology on the basis of the recent major technical improvements in surgical adhesive and gluing substances. Cyanoacrylate glue makes the underlying myocardium very stiff, noncompliant, and akinetic so that infarcted myocardium immediately surrounding the defect is strengthened. Also, because of its very high adhesive power, the patch in contact with the lesions and, consequently, the akinetic area transformed by the glue have relatively small dimensions. On the other hand, biologic glue, which is used to fix the second patch over the first one and over more distal myocardium, shows elastic and adhesive features that allow reduction of tension on the repair without impairing residual myocardium contractility in the perinfarcted area.

A possible advantage of this technique is reduced trauma to an already infarcted area because no myocardium has to be removed and no stitches have to be passed through friable tissues. Another 

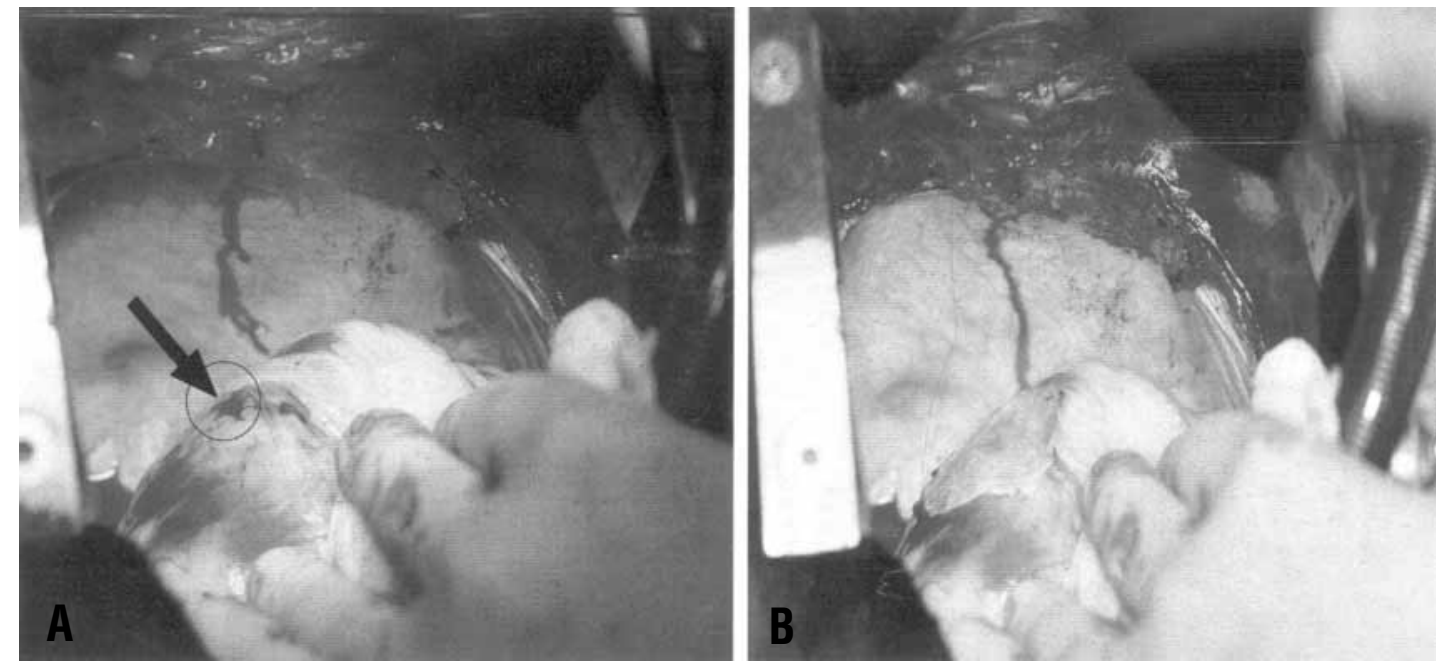

Figure 1. A, Dislocation of the heart with 2 small tissue slices and inspection of the infarcted area. B, Application of the first patch with cyanoacrylate glue.
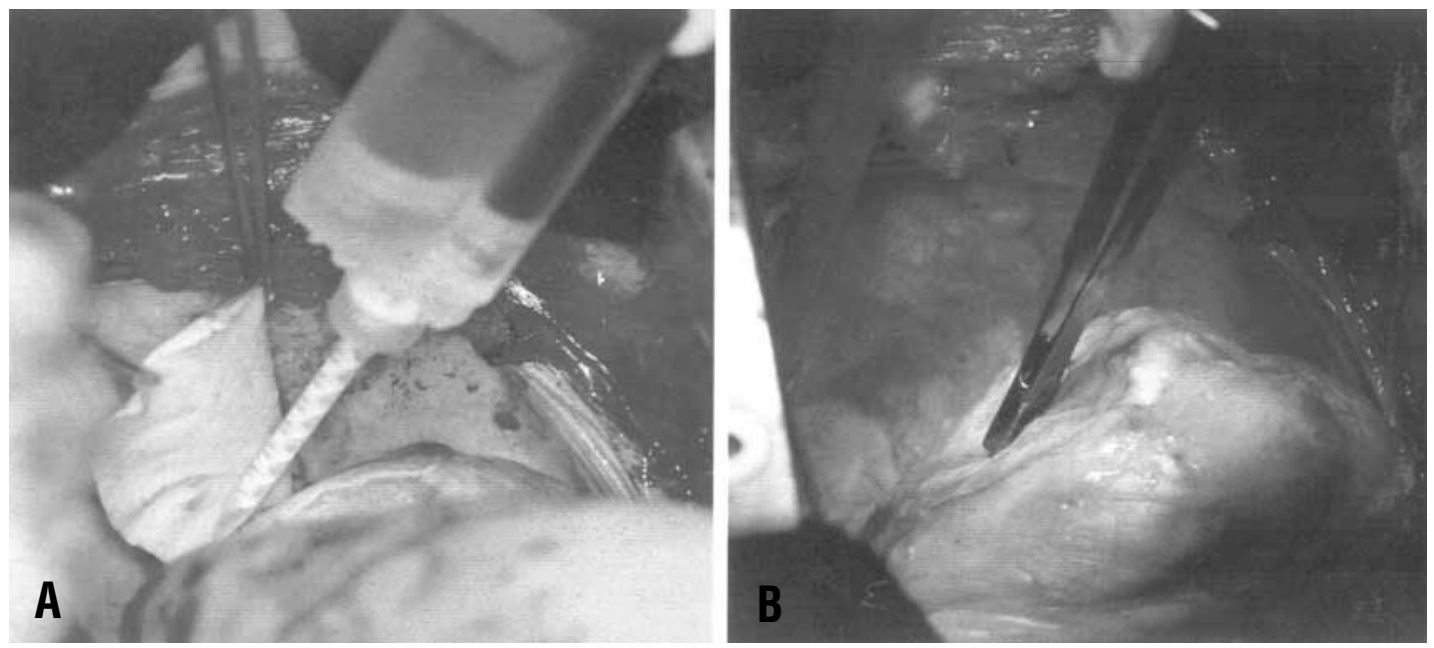

Figure 2. A, Application of biologic glue and apposition of the second sail-like patch. B, Checking of complete biologic glue polymerization after the positioning of the second patch.

advantage is lower aortic crossclamp times with respect to the techniques based on stitching patches, so that the additional ischemia to an already jeopardized myocardium is reduced.

However, because this kind of repair is possible only for the high adhesive power of cyanoacrylate glue, it is possible that this surgical technique might not be suitable for all the left ventricular free wall ruptures but only for relatively reduced dimension defects and that alternative techniques have to be adopted in larger defects. ${ }^{5}$

In conclusion, in case of relatively small left ventricular free wall ruptures, the double-patch-and-glue repair is a feasible option, although longer follow-up is needed to evaluate its success over time.

\section{References}

1. Lopez-Sendon J, Gonzalez A, Lopez de Sa E, et al. Diagnosis of subacute ventricular wall rupture after acute myocardial infarction: sensitivity and specificity of clinical, hemodynamic and echocardiographic criteria. J Am Coll Cardiol. 1992;19:1145-53.

2. Stryjer D, Friedensohn A, Hendler A. Myocardial rupture in acute myocardial infarction: urgent management. Br Heart J. 1988;59:73-4.

3. Yamazaky Y, Eguchi S, Miyamura H, et al. Replacement of myocardium with a Dacron prosthesis for complications of acute myocardial infarction. J Cardiovasc Surg (Torino). 1989;30:277-80.

4. Figueras J, Cortadellas J, Evangelista A, Soler-Soler J. Medical management of selected patients with left ventricular free wall rupture during acute myocardial infarction. J Am Coll Cardiol. 1997;29:512-8.

5. Pretre R, Benedikt P, Turina M. Experience with postinfarction left ventricular free wall rupture. Ann Thorac Surg. 2000;69:1324-5. 